\title{
SIMPLE EXAMPLE OF NONUNIQUENESS FOR A DUAL TRIGONOMETRIC SERIES
}

\author{
ROBERT B. KELMAN
}

\begin{abstract}
A simple nonzero solution is given for the classic homogeneous dual trigonometric equation having the kernel $\{\sin (n+1 / 2) x\}$. The solution's rate of growth is minimal.
\end{abstract}

Dual orthogonal series are closely connected with mixed boundary value problems. In such applications the coefficients in the orthogonal function expansions are not necessarily "small", since the functions they represent possess singularities intrinsic to the physical phenomena being modelled. As a result, uniqueness for dual expansions is of unusual practical as well as mathematical importance, and, in fact, the first example of nonuniqueness was given by Srivastav [3] in his studies of Griffith cracks. The known examples of nonuniqueness are complicated [1], [3]. In this note an observation allows us to present a simple and rigorous nonzero solution of minimal growth rate to the dual equation

$$
\begin{gathered}
\lim _{r=1-0} \sum_{n=0}^{\infty}\left[\frac{a_{n}}{n+1 / 2} \sin (n+1 / 2) x\right] r^{n}=0, \quad 0<x<c, \\
\lim _{r=1-0} \sum_{n=0}^{\infty}\left[a_{n} \sin (n+1 / 2) x\right] r^{n}=0, \quad c<x<\pi .
\end{gathered}
$$

Mathematically such examples are used in establishing sharp bounds on growth rates for coefficients needed to insure uniqueness.

In [1] it was shown that $a_{n} \equiv 0$ is the only solution to (1A, B) satisfying

$$
\sum_{0}^{N}\left|a_{n}\right|=o\left(N^{3 / 2}\right)
$$

Here we shall show that $b_{n}=(n+1 / 2) P_{n}(\cos c)$ is a solution to $(1 \mathrm{~A}, \mathrm{~B})$ where $P_{n}$ is a Legendre polynomial. Note that $\Sigma_{0}^{N}\left|b_{n}\right|=O\left(N^{3 / 2}\right)$, since $P_{n}(\cos c)=O\left(n^{-1 / 2}\right)$. Thus $\left\{b_{n}\right\}$ has minimal rate of growth.

We recall [2, p. 299] the Fourier expansion for the Lebesgue integrable function on the left:

$$
\begin{aligned}
& {[2(\cos x-\cos c)]^{-1 / 2}=\sum_{n=0}^{\infty} P_{n}(\cos c) \exp \{i(n+1 / 2) x\},} \\
& \quad 0 \leqslant x \leqslant \pi, x \neq c .
\end{aligned}
$$

Received by the editors December 15, 1978.

AMS (MOS) subject classifications (1970). Primary 42A48; Secondary 31A25, 73M05. 
Using the imaginary terms in (3) for $x \in[0, c)$ and the regularity of Abel summation we get (1A). Now use the real terms in (3) for $x \in(c, \pi]$. Applying Fatou's theorem [4, p. 99] on the Abel summability of formally differentiated Fourier series we obtain (1B).

\section{REFERENCES}

1. R. B. Kelman, A Dirichlet-Jordan theorem for dual trigonometric series, Pacific J. Math. 59 (1975), 113-123.

2. T. M. MacRobert, Spherical harmonics, 3rd ed., Pergamon Press, Oxford, New York, 1967.

3. R. P. Srivastav, Dual series relations. V. A generalized Schlömlich series and the uniqueness of the solution of dual equations involving trigonometric series, Proc. Roy. Soc. Edinburgh Sect. A 66 (1963-1964), 258-268.

4. A. Zygmund, Trigonometric series, Vol. 1, 2nd ed., Cambridge University Press, LondonNew York, 1968.

Department of Computer Science, Colorado State University, fort Coluins, ColORADO 80523 